\title{
Modeling a Three-axle Truck and Vibration Analysis under Sinusoidal Road Surface Excitation
}

\author{
Seyed Mohammad Javad Zeidi \\ PhD Candidate, Department of \\ Mechanical and Aerospace \\ Engineering, University of \\ Florida \\ Gainseville, USA
}

\author{
Pedram Hoseini \\ MSc candidate, Department of \\ Mechanical Engineering, \\ Amirkabir University of \\ Technology \\ Tehran, Iran
}

\author{
Ali Rahmani \\ Assistant professor at Shahid \\ Rajaee University \\ Department of Mechanical \\ Engineering \\ Tehran, Iran
}

\begin{abstract}
In this research, ride quality of a multi-degree of freedom 3-axle rigid truck is investigated to understand its vibration specifications. The truck is a Benz 2624 model. The system is considered for an off-road duty. Current research concentrates on the modeling and simulating the truck. The linear model indicates cab and seat suspensions, rigid live axles, and suspension geometries. The Lagrange's equation is used to obtain the motion equations and system matrices, and the numerical central difference method is utilized to obtain the system responses subject to sinusoidal road excitations. Since physical parameters of the vehicle were not available, the truck is modeled in Solidworks CAD software to obtain the dynamic properties of its components. Then, a code is developed in MATLAB to calculate system time responses under different cases for the truck moving in high speed. The developed model can also be used in newer truck with some modifications. It is also necessary to have accurate information for input data in order to change the current model.
\end{abstract}

Keywords: Vibration analysis, Lagrange equations, System response, Multi-axles truck.

\section{INTRODUCTION}

Nowadays modeling plays an important role in engineering design in various fields of engineering such as civil, materials and mechanical engineering [1-22]. Vibration modeling and analyzing is one of the application of modeling in mechanical engineering in order to enhance the design of products, e.g. aerospace [23-25], automobile and transportation [26-27]. The most common goal is identification and suppression of unwanted vibration to improve product quality. For instance, Cellular materials such as aluminum foams can be employed to dissipate vibration in vehicles. Multi-axel truck is a real world example, which requires its vibration breakdown.

Interaction between vehicles wheels and road surface causes a dynamic excitation. the vehicle speed and the elevation of the road surface unevenness causes different vibration levels [9]. Heavy vehicles are found to produce the most perceptible vibrations. The vehicle models are consisted of discrete masses, springs, friction elements and dampers which are used to describe the dynamic behavior of vehicle [10-13, 28]. When a linear model of vehicle is used, by utilizing Frequency Response Functions the calculation of axle loads is facilitated $[10,11]$. Local road unevenness is expressed by a deterministic function that shows the deviation of the travelled surface from a true planar surface. Global road unevenness can also be expressed in a stochastic way by use of a Power Spectral Density [29-34].

Many models such as quarter, bicycle, half and full models of vehicle with different numbers of DoF have been investigated in vehicle dynamics [35-38]. One of the most famous models for vehicles is eight-DoF model, including forward, lateral, yaw and rolling motion plus, four degree of freedom for travel of each wheel [39-40]. Multibody system dynamic models of vehicles have also been proposed in the literature. For instance, Rahmani Hanzaki et al. proposed a methodology for dynamic analysis of a multibody system with spherical joints. They considered a suspension system of a vehicle as an example for that [41]. Applying this methodology on a threeaxle truck complicates the calculation. Hence, other discrete models were employed for these trucks. For example, Tabatabaee developed a 16-DoF non-linear model for an articulated vehicle, which is validated experimentally [42]. It is also possible to reduce air pollutants specially $\mathrm{CO} 2$ by acquisition of developed model by optimizing several components in the truck similar to the work done for the other systems which were successful. This paper presents a survey on the equations of motion utilizing Lagrange equations to determine system responses subject to sinusoidal road excitations of a complete 3-D rigid three-axle truck model, i.e., Benz 2624 model. This analysis is helpful for better understanding of the coupled motions of the wheels. The validation of our equations have been verified with ADAMS in our previous work [43]. The developed $19 \mathrm{DoF}$ model can also be applied on many trucks by changing material properties and adding estimations. Previous defined equations has also been very useful in the current paper. Mathematical model which was developed and derived by Zeidi et al. is used during the current mathematical modeling and code writing in MATLAB [44-50].

\section{MODELING THE THREE-AXLE TRUCK}

Using experimental techniques to obtain mass properties of the components of a manufactured vehicle is reasonable but very costly; hence, in this work, Solidworks software is employed to model a three-axle truck and to find masses, centers of mass, moments of inertia etc. These physical properties are highly necessary for dynamic simulation of the truck. Figures 1 and 2 show two views of the assembled model of the truck, and some components of the truck, respectively. In this part, the weighty components of the truck such as chassis, tires, differentials, cabin, springs etc. are 
modeled precisely. Non homogeneous material is assigned to this model since different material was utilized in the current model and it was very important to have a very precise model.

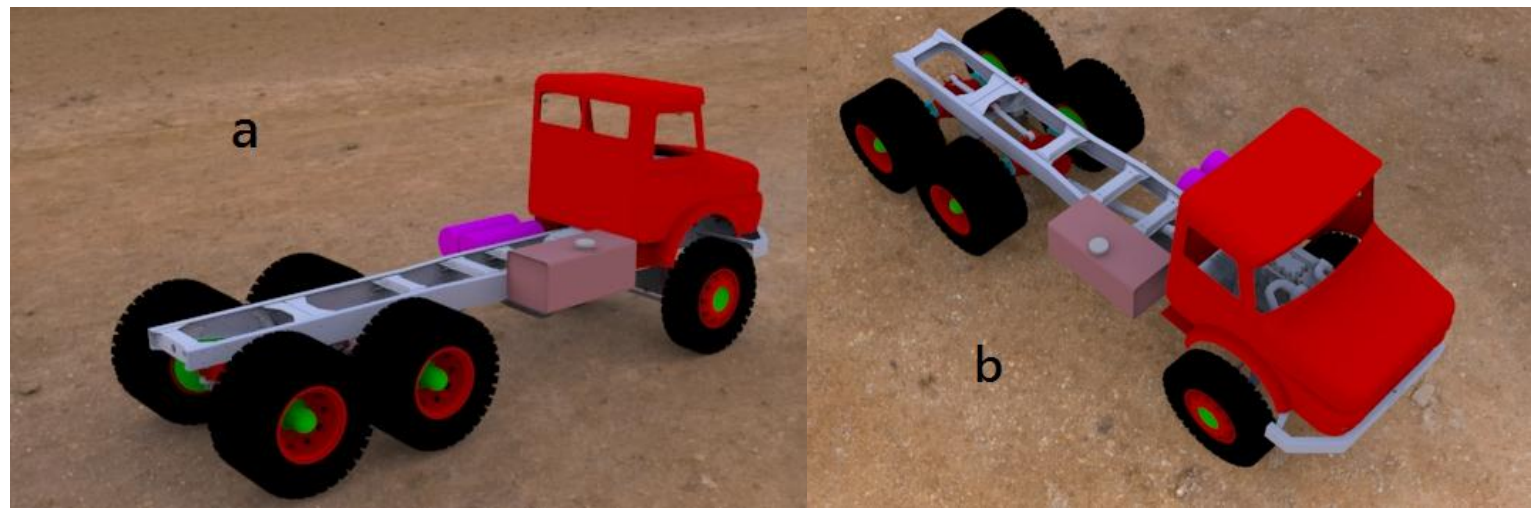

Figure 1. Two views of the CAD model from the three-axle truck;

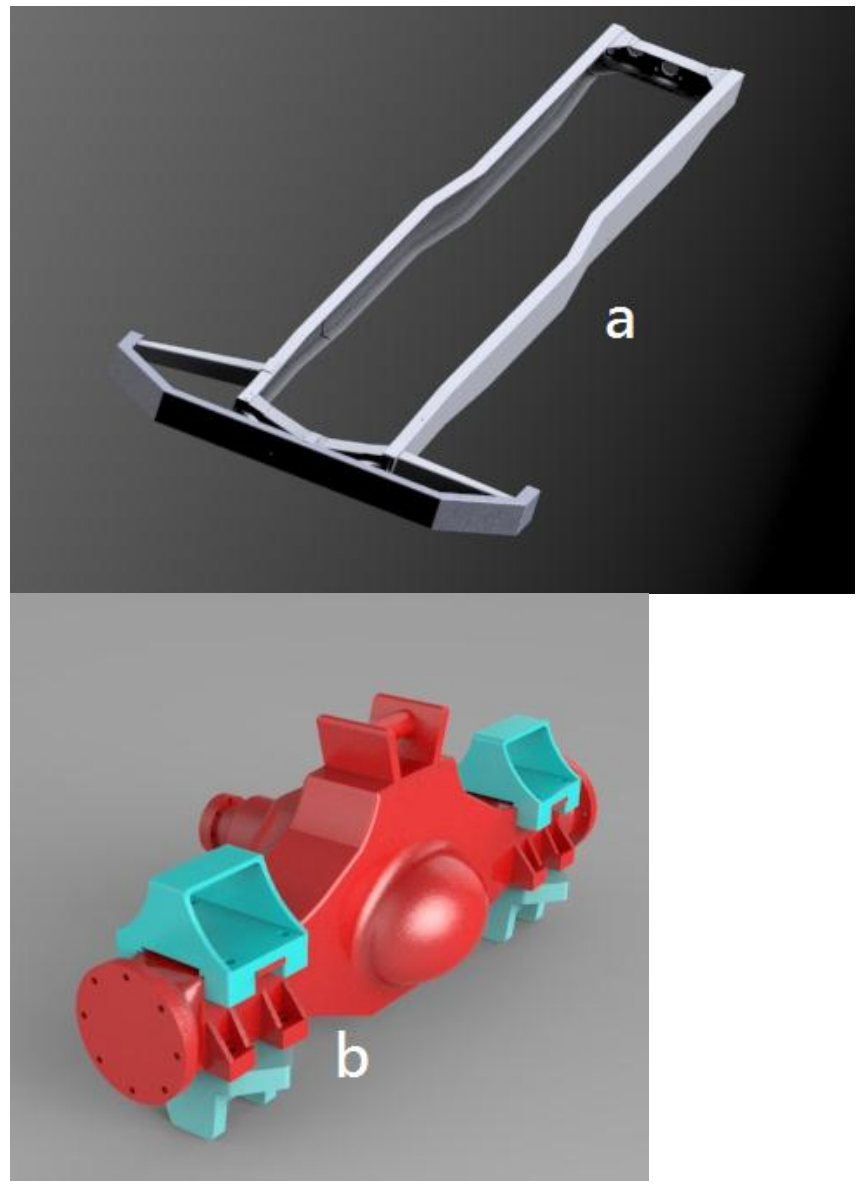

Figure 2. CAD models of two main components of the truck; a) chassis, b) axle with differential

\section{GOVERNING EQUATIONS}

Lagrange method is utilized to determine dynamic behavior of the mentioned three-axle truck. The truck is considered as a 19-DoF mathematical model. As shown in Figure 3. $\mathrm{M}_{1}, \mathrm{M}_{2}$ and $\mathrm{M}_{3}$ are the axles of the truck.
Blue springs are considered on behalf of tires, red springs as leaf springs of the suspensions systems. Green springs are counted for connecting cabin to the frame and finally, purple spring is used to suspend driver's seat with respect to the cabin. As the rests, $W$, $\theta$, and $\varphi$ illustrate displacement, roll, and pitch of the truck in this dynamic analysis. Hence, the 19 DoFs are as follow:

- Driver seat bounce, one degree; $w_{106}$;

- Cab bounce, pitch and roll, three degrees; orderly $w_{104}, \theta_{104}, \varphi_{104}$;

- Chassis bounce (sprung mass), pitch and roll, three degrees; $w_{100}, \varphi_{100}, \theta_{100}$, respectively:

- Front axle, its bounce and roll, two degrees; orderly $w_{101}, \theta_{101}$;

- Intermediate axle, bounce and roll, two degrees; orderly $w_{102}, \theta_{102}$;

- Rear axle, bounce and roll, , two degrees; orderly $w_{103}, \theta_{103}$;

- 6 bounce motion of the 6 wheels; $w_{1}, w_{2}, w_{3}$, $\mathrm{w}_{4}, \mathrm{w}_{5}, \mathrm{w}_{6} ;$ where $\mathrm{w}_{1}$ and $; \mathrm{w}_{2}$ are the bounce of left and right steer wheels, respectively; $w_{3}$ and $w_{4}$ are the bounce of left and right wheels of the middle axle, correspondingly; $w_{5}$ and $w_{6}$ are the bounce of left and right wheels of rear axle, respectively.

The vector of coordinates for the vehicle is written as:

$W_{19}=$

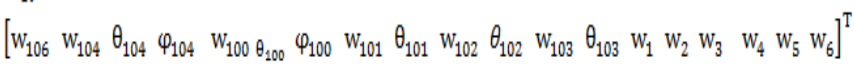

(1) 


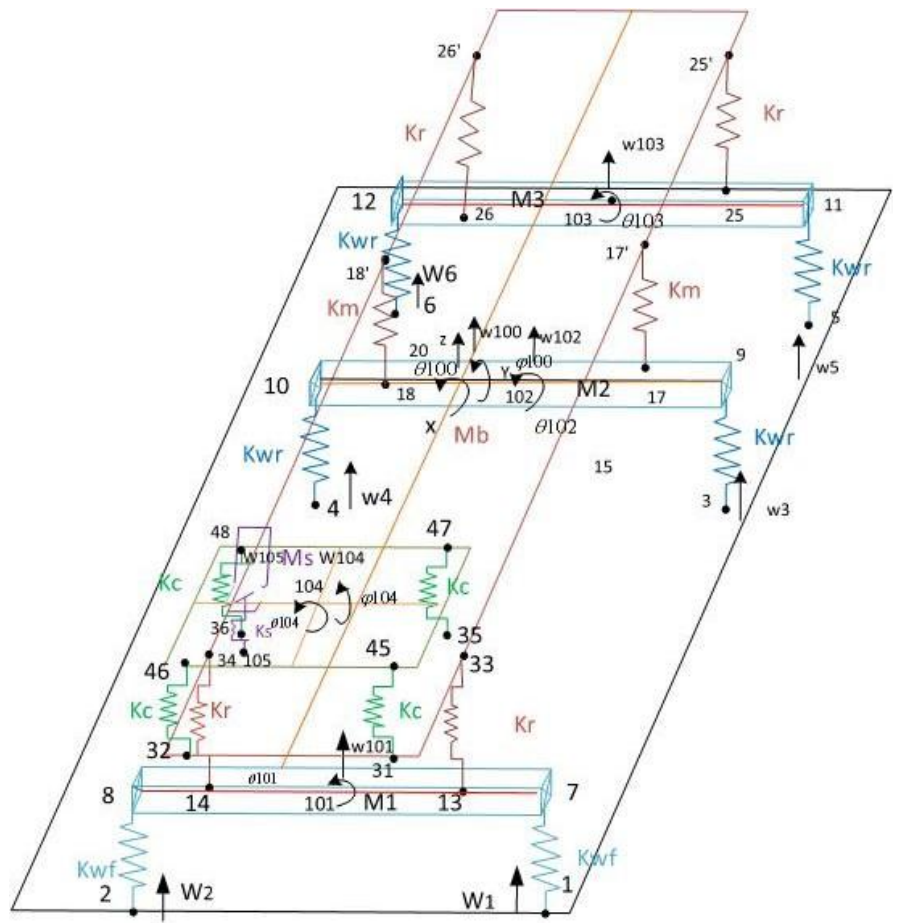

Figure 3. The scheme of the 19-DoF model for the truck

Figure 4(a) shows truck model in $\mathrm{X}-\mathrm{Z}$ plane and distances between different important points. In addition, Figure 4(b) indicates the model in $\mathrm{Y}-\mathrm{Z}$ plane and the related parameters.

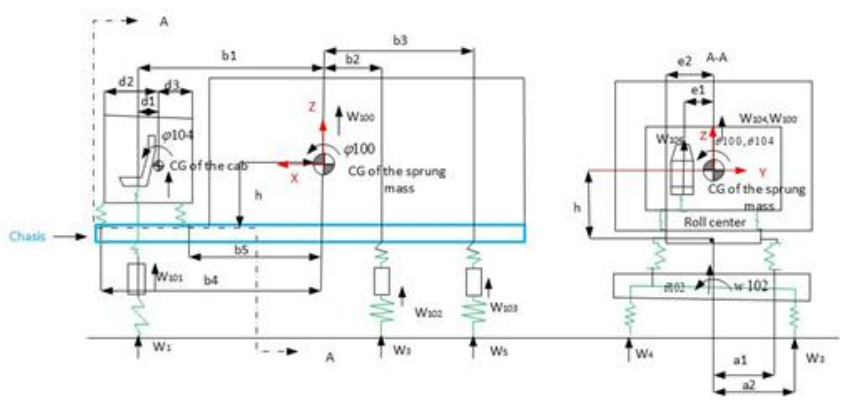

Figure 4. CGs (Center of gravity) and other essential distances of the model

\section{EQUATIONS OF MOTION}

The Lagrange equation is well-known in the following form for this system:

$\frac{d}{d t}\left(\frac{d T}{d \dot{W}_{19}}\right)-\left(\frac{d T}{d W_{19}}\right)+\left(\frac{d P}{d W_{19}}\right)+\left(\frac{d R}{d \dot{W}_{19}}\right)=0$

(2)

where $\mathrm{T}, \mathrm{P}$ and $\mathrm{R}$ are the kinematic, potential and dissipation energies of the system, respectively.

The kinetic energy of the system is as follow:

$$
\begin{aligned}
& \mathrm{T}=\frac{1}{2} \mathrm{M}_{\mathrm{s}}\left(\mathrm{W}^{\prime} 106\right)^{2}+\frac{1}{2} \mathrm{M}_{\mathrm{c}}\left(\mathrm{W}^{\prime} 104\right)^{2}+ \\
& \frac{1}{2} \mathrm{M}_{\mathrm{b}}\left(\mathrm{W}^{\prime} 100\right)^{2}+\frac{1}{2} \mathrm{M}_{1}\left(\mathrm{~W}^{\prime} 101\right)^{2}+ \\
& \frac{1}{2} \mathrm{M}_{2}\left(\mathrm{~W}^{\prime} 102\right)^{2}+\frac{1}{2} \mathrm{M}_{3}\left(\mathrm{~W}^{\prime} 103\right)^{2}+ \\
& \frac{1}{2} \mathrm{I}_{\mathrm{cx}}\left(\theta^{\prime} 104\right)^{2}+\frac{1}{2} \mathrm{I}_{\mathrm{bx}}\left(\theta^{\prime} 100\right)^{2}+\frac{1}{2} \mathrm{I}_{1 \mathrm{x}}\left(\theta^{\prime} 101\right)^{2}+ \\
& \frac{1}{2} \mathrm{I}_{2 \mathrm{x}}\left(\theta^{\prime} 102\right)^{2}+\frac{1}{2} \mathrm{I}_{3 \mathrm{x}}\left(\theta^{\prime} 103\right)^{2}+\frac{1}{2} \mathrm{I}_{\mathrm{cy}}\left(\theta^{\prime} 104\right)^{2}+ \\
& \frac{1}{2} \mathrm{I}_{\mathrm{by}}\left(\theta^{\prime} 100\right)^{2}
\end{aligned}
$$

Moreover, the potential energy of the system is obtained as:

$\mathrm{P}=\frac{1}{2} \mathrm{~K}_{\mathrm{s}}(\mathrm{W} 106-\mathrm{W} 105)^{2}+\frac{1}{2} \mathrm{~K}_{\mathrm{c}}(\mathrm{W} 45-$

$\mathrm{W} 31)^{2}+\frac{1}{2} \mathrm{~K}_{\mathrm{c}}(\mathrm{W} 46-\mathrm{W} 32)^{2}+\frac{1}{2} \mathrm{~K}_{\mathrm{c}}(\mathrm{W} 47-$

$\mathrm{W} 35)^{2}+\frac{1}{2} \mathrm{~K}_{\mathrm{c}}(\mathrm{W} 48-\mathrm{W} 36)^{2}+\frac{1}{2} \mathrm{~K}_{f}(\mathrm{~W} 33-$

$\mathrm{W} 13)^{2}+\frac{1}{2} \mathrm{~K}_{f}(\mathrm{~W} 34-\mathrm{W} 14)^{2}+\frac{1}{2} \mathrm{~K}_{m}\left(\mathrm{~W} 17^{-}-\right.$

$\mathrm{W} 17)^{2}+\frac{1}{2} \mathrm{~K}_{m}\left(\mathrm{~W}_{18}{ }^{-}-\mathrm{W} 18\right)^{2}+\frac{1}{2} \mathrm{~K}_{r}\left(\mathrm{~W} 25^{`}-\right.$

$\mathrm{W} 25)^{2}+\frac{1}{2} \mathrm{~K}_{r}(\mathrm{~W} 26-\mathrm{W} 26)^{2}+\frac{1}{2} \mathrm{~K}_{\mathrm{Wf}}(\mathrm{W} 7-$

$\mathrm{W} 1)^{2}+\frac{1}{2} \mathrm{~K}_{\mathrm{Wf}}(\mathrm{W} 8-\mathrm{W} 2)^{2}+\frac{1}{2} \mathrm{~K}_{\mathrm{Wr}}(\mathrm{W} 9-$

$W 3)^{2}+\frac{1}{2} K_{W r}(W 10-W 4)^{2}+\frac{1}{2} K_{W r}(W 11-$

$\mathrm{W} 5)^{2}+\frac{1}{2} \mathrm{~K}_{\mathrm{Wr}}(\mathrm{W} 12-\mathrm{W} 6)^{2}$

And the dissipation energy of the system is:

$\mathrm{R}=\frac{1}{2} \mathrm{c}_{\mathrm{s}}(\dot{\mathrm{W}} 106-\dot{\mathrm{W}} 105)^{2}+\frac{1}{2} \mathrm{c}_{\mathrm{c} 1}(\dot{\mathrm{W}} 45-$ $\dot{W} 31)^{2}+\frac{1}{2} c_{\mathrm{c} 2}(\dot{W} 46-\dot{W} 32)^{2}+\frac{1}{2} c_{\mathrm{c} 3}(\dot{W} 47-$ $\dot{W} 35)^{2}+\frac{1}{2} c_{c 4}(\dot{W} 48-\dot{W} 36)^{2}+\frac{1}{2} c_{1}(\dot{W} 33-$ $\dot{W} 13)^{2}+\frac{1}{2} c_{2}(\dot{W} 34-\dot{W} 14)^{2}+\frac{1}{2} c_{e 3}\left(\dot{W} 17^{-}-\right.$ $\dot{W} 17)^{2}+\frac{1}{2} c_{e 4}\left(\dot{W} 18^{\circ}-\dot{W} 18\right)^{2}+\frac{1}{2} c_{e 5}\left(\dot{W} 25^{\circ}-\right.$ $\dot{W} 25)^{2}+\frac{1}{2} c_{e 6}(\dot{W} 26-\dot{W} 26)^{2}$

By differentiating of $\mathrm{T}, \mathrm{P}$ and $\mathrm{R}$ with respect to the coordinates and time according to eq. (2), equations of motion can be organized as:

$M \cdot \ddot{W}+C \cdot \dot{W}+K \cdot W=0$ 
In which $\mathrm{M}_{19}, \mathrm{~K}_{19}$ and $\mathrm{C}_{19}$ are orderly mass matrix, stiffness matrix and damping matrix of the 19 DoF of the truck-poster system model. In this equation, $\ddot{W}_{19}, \dot{W}_{19}$ and $W_{19}$ are acceleration vector, velocity vector and displacement vector of the 19 DoF truckposter system model. In addition, system mass matrix, $\mathrm{M}_{19}$ which is a diagonal matrix, is calculated as follow:

$M_{19}=\operatorname{diag}\left[M_{s} M_{c} I_{c x} I_{c y} M_{b} I_{b x} I_{b y} M_{1} I_{1 x} M_{2} I_{2 x} M_{3}\right.$

Where, "diag" illustrates that the $\mathrm{M}_{19}$ is a diagonal matrix and $\mathrm{M}_{\mathrm{s}}$ to $\mathrm{M}_{06}$ are located on the main diagonal of the matrix. In this relation, $\mathrm{M}_{\mathrm{s}}$ and $\mathrm{M}_{\mathrm{c}}$ are masses of the seat and the driver, and the cab, respectively; $\mathrm{I}_{\mathrm{cx}}$ and $\mathrm{I}_{\mathrm{cy}}$ are inertia of the cab about $\mathrm{X}$ and $\mathrm{Y}$ axes, correspondingly. In the following, $\mathrm{M}_{\mathrm{b}}, \mathrm{I}_{\mathrm{bx}}$, and $\mathrm{I}_{\mathrm{by}}$ point to sprung mass, inertia of the sprung mass about $\mathrm{X}$ and $\mathrm{Y}$ axes, respectively; Also, 1, 2, and 3 as the indexes in order point to the front axle, middle axle, and the rear axle of the truck. Similarly, $M_{01}$ to $M_{06}$ indicate the masses of the front left to rear right wheels, as well. The 19 nonzero values have been obtained from the truck model in Solidworks software utilizing mass properties. Now, the system damping matrix and stiffness matrix can be written in the following form:

$C_{19}=\left[\begin{array}{cccc}C_{1,1} & C_{1,2} & \ldots & C_{1,19} \\ C_{2,1} & C_{2,2} & & C_{2,19} \\ & \vdots & \ddots & \vdots \\ C_{19,1} & C_{19,2} & \cdots & C / 19,19\end{array}\right], \quad K_{19}=\left[\begin{array}{cccc}K_{1,1} & K_{1,2} & & K_{1,19} \\ K_{2,1} & K_{2,2} & \cdots & K_{2,19} \\ \vdots & & \ddots & \vdots \\ K_{19,1} & K_{19,2} & \cdots & K_{19,19}\end{array}\right]$

The non-zero components of $C_{19}$ and $K_{19}$ are as follows:

$$
\begin{aligned}
& \mathrm{C}_{1,1}=\mathrm{C}_{\mathrm{s}}, \mathrm{C}_{1,2}=\mathrm{C}_{2,1}=-\mathrm{C}_{\mathrm{s}}, \mathrm{C}_{1,3}=\mathrm{C}_{3,1}= \\
& \mathrm{C}_{\mathrm{s}} \cdot \mathrm{e}_{1}, \mathrm{C}_{1,4}=\mathrm{C}_{4,1}=\mathrm{C}_{\mathrm{s}} \cdot \mathrm{d}_{1} \\
& ; \\
& \mathrm{C}_{2,2}=\mathrm{C}_{\mathrm{s}}+\mathrm{C}_{c 1}+\mathrm{C}_{c 2}+\mathrm{C}_{c 3}+\mathrm{C}_{c 4}, \\
& \mathrm{C}_{2,3}=\mathrm{C}_{3,2}=-\mathrm{C}_{\mathrm{s}} \cdot \mathrm{e}_{1}+\mathrm{C}_{\mathrm{c} 1} \cdot \mathrm{e}_{2}-\mathrm{C}_{\mathrm{c} 2} \cdot \mathrm{e}_{2}+ \\
& \mathrm{C}_{\mathrm{c} 3} \cdot \mathrm{e}_{2}-\mathrm{C}_{\mathrm{c} 4} \cdot \mathrm{e}_{2}
\end{aligned}
$$$$
\mathrm{C}_{2,4}=\mathrm{C}_{4,2}=-\mathrm{C}_{\mathrm{s}} \cdot \mathrm{d}_{1}-\mathrm{C}_{\mathrm{c} 1} \cdot \mathrm{d}_{2}-\mathrm{C}_{4} \cdot \mathrm{C}_{2} \cdot \mathrm{d}_{2}+
$$$$
\mathrm{C}_{\mathrm{c} 3} \cdot \mathrm{d}_{3}+\mathrm{C}_{\mathrm{c} 4} \cdot \mathrm{d}_{3}
$$$$
\text { , } \mathrm{C}_{2,5}=\mathrm{C}_{5,2}=-\mathrm{C}_{c 1}-\mathrm{C}_{c 2}-\mathrm{C}_{c 3}-\mathrm{C}_{c 4} \text {, }
$$$$
\mathrm{C}_{2,6}=\mathrm{C}_{6,2}=\mathrm{C}_{3,5}=\mathrm{C}_{5,3}=-\mathrm{C}_{\mathrm{c} 1} \cdot \mathrm{e}_{2}+\mathrm{C}_{\mathrm{c} 2} \cdot \mathrm{e}_{2}-
$$$$
\mathrm{C}_{\mathrm{c} 3} \cdot \mathrm{e}_{2}+\mathrm{C}_{\mathrm{c} 4} \cdot \mathrm{e}_{2}
$$

$$
\begin{aligned}
& \mathrm{C}_{2,7}=\mathrm{C}_{7,2}=\mathrm{C}_{\mathrm{c} 1} \cdot \mathrm{b}_{4}+\mathrm{C}_{\mathrm{c} 2} \cdot \mathrm{b}_{4}+\mathrm{C}_{\mathrm{c} 3} \cdot \mathrm{b}_{5}+ \\
& \mathrm{C}_{\mathrm{c} 4} \cdot \mathrm{b}_{5} ;
\end{aligned}
$$

$$
\begin{aligned}
& \mathrm{C}_{3,3}=\mathrm{C}_{s} \cdot \mathrm{e}_{1}^{2}+\mathrm{C}_{\mathrm{c} 1} \cdot \mathrm{e}_{2}^{2}+\mathrm{C}_{\mathrm{c} 2} \cdot \mathrm{e}_{2}^{2}+\mathrm{C}_{\mathrm{c} 3} \cdot \mathrm{e}_{2}^{2}+ \\
& \mathrm{C}_{\mathrm{c} 4} \cdot \mathrm{e}_{2}^{2} \\
& \mathrm{C}_{3,4}=\mathrm{C}_{4,3}=\mathrm{C}_{\mathrm{s}} \cdot \mathrm{d}_{1} \cdot \mathrm{e}_{1}-\mathrm{C}_{\mathrm{c} 1} \cdot \mathrm{d}_{2} \cdot \mathrm{e}_{2}+
\end{aligned}
$$

;

$$
\begin{aligned}
& \mathrm{C}_{3,6}=\mathrm{C}_{6,3}=-\mathrm{C}_{\mathrm{c} 1} \cdot \mathrm{e}_{2}^{2}-\mathrm{C}_{\mathrm{c} 2} \cdot \mathrm{e}_{2}^{2}-\mathrm{C}_{\mathrm{c} 3} \cdot \mathrm{e}_{2}^{2}- \\
& \mathrm{C}_{\mathrm{c} 4} \cdot \mathrm{e}_{2}^{2} \\
& \mathrm{C}_{3,7}=\mathrm{C}_{7,3}=\mathrm{C}_{\mathrm{c} 1} \cdot \mathrm{b}_{4} \cdot \mathrm{e}_{2}-\mathrm{C}_{\mathrm{c} 2} \cdot \mathrm{b}_{4} \cdot \mathrm{e}_{2}+ \\
& \mathrm{C}_{\mathrm{c} 3} \cdot \mathrm{b}_{5} \cdot \mathrm{e}_{2}-\mathrm{C}_{\mathrm{c} 4} \cdot \mathrm{b}_{5} \cdot \mathrm{e}_{2} \\
& ; \\
& \mathrm{C}_{4,4}=\mathrm{C}_{s} \cdot \mathrm{d}_{1}^{2}+\mathrm{C}_{\mathrm{c} 1} \cdot \mathrm{d}_{2}^{2}+\mathrm{C}_{\mathrm{c} 2} \cdot \mathrm{d}_{2}^{2}+\mathrm{C}_{\mathrm{c} 3} \cdot \mathrm{d}_{3}^{2}+ \\
& \mathrm{C}_{\mathrm{c} 4} \cdot \mathrm{d}_{3}^{2} \\
& \mathrm{C}_{4,5}=\mathrm{C}_{5,4}=\mathrm{C}_{\mathrm{c} 1} \cdot \mathrm{d}_{2}+\mathrm{C}_{\mathrm{c} 2} \mathrm{~d}_{2}-\mathrm{C}_{\mathrm{c} 3} \cdot \mathrm{d}_{3}-\mathrm{C}_{\mathrm{c} 4} \cdot \mathrm{d}_{3}, \\
& \mathrm{C}_{4,6}=\mathrm{C}_{6,4}=\mathrm{C}_{\mathrm{c} 1} \cdot \mathrm{d}_{2} \cdot \mathrm{e}_{2}-\mathrm{C}_{\mathrm{c} 2} \cdot \mathrm{d}_{2} \cdot \mathrm{e}_{2}- \\
& \mathrm{C}_{\mathrm{c} 3} \cdot \mathrm{d}_{3} \cdot \mathrm{e}_{2}+\mathrm{C}_{\mathrm{c} 4} \cdot \mathrm{d}_{3} \cdot \mathrm{e}_{2} \\
& \mathrm{C}_{4,7}=\mathrm{C}_{7,4}=-\mathrm{C}_{\mathrm{c} 1} \cdot \mathrm{b}_{4} \cdot \mathrm{d}_{2}-\mathrm{C}_{\mathrm{c} 2} \cdot \mathrm{b}_{4} \cdot \mathrm{d}_{2}+ \\
& \mathrm{C}_{\mathrm{c} 3} \cdot \mathrm{b}_{5} \cdot \mathrm{d}_{3}+\mathrm{C}_{\mathrm{c} 4} \cdot \mathrm{b}_{5} \cdot \mathrm{d}_{3} ;
\end{aligned}
$$

$$
\begin{aligned}
& \mathrm{C}_{5,5}=\mathrm{C}_{c 1}+\mathrm{C}_{c 2}+\mathrm{C}_{c 3}+\mathrm{C}_{c 4}+\mathrm{C}_{1}+\mathrm{C}_{2}+ \\
& \mathrm{C}_{3} \cdot l_{2}^{2} / l_{1}+\mathrm{C}_{4} \cdot l_{2}^{2} / l_{1}+\mathrm{C}_{5} \cdot l_{2}^{2} / l_{1}+\mathrm{C}_{6} \cdot l_{2}^{2} / l_{1}, \\
& \mathrm{C}_{5,6}=\mathrm{C}_{6,5}=\mathrm{C}_{c 1} \cdot e_{2}-\mathrm{C}_{c 2} \cdot e_{2}+\mathrm{C}_{c 3} \cdot e_{2}- \\
& \mathrm{C}_{c 4} \cdot e_{2}+\mathrm{C}_{1} \cdot a_{1}-\mathrm{C}_{2} \cdot a_{1}+\mathrm{C}_{3} \cdot l_{2}^{2} / l_{1} \cdot a_{1}- \\
& \mathrm{C}_{4} \cdot l_{2}^{2} / l_{1} \cdot a_{1}+\mathrm{C}_{5} \cdot l_{2}^{2} / l_{1} \cdot a_{1}-\mathrm{C}_{6} \cdot l_{2}^{2} / l_{1} \cdot a_{1} ; \\
& \mathrm{C}_{5,7}=\mathrm{C}_{7,5}=-\mathrm{C}_{c 1} \cdot b_{4}-\mathrm{C}_{c 2} \cdot b_{4}-\mathrm{C}_{c 3} \cdot b_{5}- \\
& \mathrm{C}_{1} \cdot b_{1}-\mathrm{C}_{2} \cdot b_{1}+\mathrm{C}_{3} \cdot l_{2}^{2} / l_{1} \cdot b_{2}+\mathrm{C}_{4} \cdot l_{2}^{2} / l_{1} \cdot b_{2} ; \\
& \mathrm{C}_{5,8}=\mathrm{C}_{8,5}=-\mathrm{C}_{1}-\mathrm{C}_{2}, \\
& \mathrm{C}_{5,9}=\mathrm{C}_{9,5}=\mathrm{C}_{6,8}=\mathrm{C}_{8,6}=-\mathrm{C}_{1} \cdot a_{1}+\mathrm{C}_{2} \cdot a_{1}, \\
& \mathrm{C}_{5,10}=\mathrm{C}_{10,5}=-\mathrm{C}_{3} \cdot l_{2}^{2} / l_{1}-\mathrm{C}_{4} \cdot l_{2}^{2} / l_{1} ; \\
& \mathrm{C}_{5,11}=\mathrm{C}_{11,5}=\mathrm{C}_{6,10}=\mathrm{C}_{10,6}=-\mathrm{C}_{3} \cdot l_{2}^{2} / l_{1} \cdot a_{1}+ \\
& \mathrm{C}_{4} \cdot l_{2}^{2} / l_{1} \cdot a_{1}
\end{aligned}
$$


$\mathrm{C}_{5,12}=\mathrm{C}_{12,5}=\mathrm{C}_{6,12}=\mathrm{C}_{12,6}=-\mathrm{C}_{5} \cdot l_{2}^{2} / l_{1}+$

$\mathrm{C}_{6} \cdot l_{2}^{2} / l_{1}$;

$\mathrm{C}_{5,13}=\mathrm{C}_{13,5}=-\mathrm{C}_{5} \cdot l_{2}^{2} / l_{1} \cdot a_{1}+\mathrm{C}_{6} \cdot l_{2}^{2} / l_{1} \cdot a_{1}$,

$\mathrm{C}_{6,6}=\mathrm{C}_{\mathrm{c} 1} \cdot \mathrm{e}_{2}^{2}+\mathrm{C}_{\mathrm{c} 2} \cdot \mathrm{e}_{2}^{2}+\mathrm{C}_{\mathrm{c} 3} \cdot \mathrm{e}_{2}^{2}+\mathrm{C}_{\mathrm{c} 4} \cdot \mathrm{e}_{2}^{2}+$

$\mathrm{C}_{1} \cdot a_{1}^{2}+\mathrm{C}_{2} \cdot a_{1}^{2}+\mathrm{C}_{3} \cdot l_{2}^{2} / l_{1} \cdot a_{1}^{2}+\mathrm{C}_{4} \cdot l_{2}^{2} / l_{1} \cdot a_{1}^{2}+$

$\mathrm{C}_{5} \cdot l_{2}^{2} / l_{1} \cdot a_{1}^{2}+\mathrm{C}_{6} \cdot l_{2}^{2} / l_{1} \cdot a_{1}^{2}$;

$\mathrm{C}_{6,7}=\mathrm{C}_{7,6}=-\mathrm{C}_{\mathrm{c} 1} \cdot \mathrm{b}_{4} \cdot \mathrm{e}_{2}+\mathrm{C}_{\mathrm{c} 2} \cdot \mathrm{b}_{4} \cdot \mathrm{e}_{2}-$

$\mathrm{C}_{\mathrm{c} 3} \cdot \mathrm{b}_{5} \cdot \mathrm{e}_{2}+\mathrm{C}_{\mathrm{c} 4} \cdot \mathrm{b}_{5} \cdot e_{2}-\mathrm{C}_{1} \cdot \mathrm{a}_{1} \cdot \mathrm{b}_{1}+\mathrm{C}_{2} \cdot \mathrm{a}_{1} \cdot \mathrm{b}_{1}+$

$\mathrm{C}_{3}, l_{2}^{2} / l_{1} \cdot b_{2}, a_{1}$

$-\mathrm{C}_{4} \cdot l_{2}^{2} / l_{1} \cdot b_{2} \cdot a_{1}+\mathrm{C}_{5} \cdot l_{2}^{2} / l_{1} \cdot a_{1}-\mathrm{C}_{6} \cdot l_{2}^{2} / l_{1} \cdot a_{1}$;

$\mathrm{C}_{6,9}=\mathrm{C}_{9,6}=-\mathrm{C}_{1} \cdot a_{1}^{2}-\mathrm{C}_{2} \cdot a_{1}^{2}$,

$\mathrm{C}_{6,11}=\mathrm{C}_{11,6}=-\mathrm{C}_{3} \cdot l_{2}^{2} / l_{1} \cdot a_{1}^{2}-\mathrm{C}_{4} \cdot l_{2}^{2} / l_{1} \cdot a_{1}^{2}$,

$\mathrm{C}_{6,13}=\mathrm{C}_{13,6}=-\mathrm{C}_{5} \cdot l_{2}^{2} / l_{1} \cdot a_{1}^{2}-\mathrm{C}_{6} \cdot l_{2}^{2} / l_{1} \cdot a_{1}^{2}$,

$\mathrm{C}_{7,7}=\mathrm{C}_{\mathrm{c} 1} \cdot \mathrm{b}_{4}^{2}+\mathrm{C}_{\mathrm{c} 2} \cdot \mathrm{b}_{4}^{2}+\mathrm{C}_{\mathrm{c} 3} \cdot \mathrm{b}_{5}^{2}+\mathrm{C}_{\mathrm{c} 4} \cdot \mathrm{b}_{5}^{2}+$

$\mathrm{C}_{1} \cdot b_{1}^{2}+\mathrm{C}_{2} \cdot b_{1}^{2}+\mathrm{C}_{3} \cdot l_{2}^{2} / l_{1} \cdot b_{2}^{2}+\mathrm{C}_{4} \cdot l_{2}^{2} / l_{1} \cdot b_{2}^{2}+$

$\mathrm{C}_{5} \cdot l_{2}^{2} / l_{1} \cdot b_{3}^{2}$

$+\mathrm{C}_{6} \cdot l_{2}^{2} / l_{1} \cdot b_{3}^{2}$;

$\mathrm{C}_{7,8}=\mathrm{C}_{8,7}=\mathrm{C}_{1} \cdot b_{1}+\mathrm{C}_{2} \cdot b_{1}$,

$\mathrm{C}_{7,9}=\mathrm{C}_{9,7}=\mathrm{C}_{1}, a_{1} \cdot b_{1}-\mathrm{C}_{2}, a_{1} \cdot b_{1}$,

$\mathrm{C}_{7,10}=\mathrm{C}_{10,7}=-\mathrm{C}_{3} \cdot l_{2}^{2} / l_{1} \cdot b_{2}-\mathrm{C}_{4} \cdot l_{2}^{2} / l_{1} \cdot b_{2}$,

$\mathrm{C}_{7,11}=\mathrm{C}_{11,7}=-\mathrm{C}_{3} \cdot l_{2}^{2} / l_{1} \cdot b_{2}, a_{1}+\mathrm{C}_{4} \cdot l_{2}^{2} /$

$l_{1}, b_{2}, a_{1}$

, $\mathrm{C}_{7,12}=\mathrm{C}_{12,7}=-\mathrm{C}_{5} \cdot l_{2}^{2} / l_{1} \cdot b_{3}-\mathrm{C}_{6} \cdot l_{2}^{2} / l_{1} \cdot b_{3}$,

$\mathrm{C}_{7,13}=\mathrm{C}_{13,7}=-\mathrm{C}_{5} \cdot l_{2}^{2} / l_{1} \cdot b_{3} \cdot a_{1}+\mathrm{C}_{6} \cdot l_{2}^{2} /$

$l_{1}, b_{3}, a_{1}$

$, \mathrm{C}_{8,8}=\mathrm{C}_{1}+\mathrm{C}_{2}, \mathrm{C}_{8,9}=\mathrm{C}_{9,8}=\mathrm{C}_{1} \cdot a_{1}-\mathrm{C}_{2} \cdot a_{1}$;

$\mathrm{C}_{9,9}=\mathrm{C}_{9,8}=\mathrm{C}_{1} \cdot a_{1}^{2}+\mathrm{C}_{2} \cdot a_{1}^{2}$,

$\mathrm{C}_{10,10}=\mathrm{C}_{3} \cdot l_{2}^{2} / l_{1}+\mathrm{C}_{4} \cdot l_{2}^{2} / l_{1}, \mathrm{C}_{10,11}=\mathrm{C}_{11,10}=$ $\mathrm{C}_{3} \cdot l_{2}^{2} / l_{1} \cdot a_{1}-\mathrm{C}_{4} \cdot l_{2}^{2} / l_{1} \cdot a_{1}$;

$\mathrm{C}_{11,11}=\mathrm{C}_{3} \cdot l_{2}^{2} / l_{1} \cdot a_{1}^{2}+\mathrm{C}_{4} \cdot l_{2}^{2} / l_{1} \cdot a_{1}^{2}$,

$\mathrm{C}_{12,12}=\mathrm{C}_{5} \cdot l_{2}^{2} / l_{1}+\mathrm{C}_{6} \cdot l_{2}^{2} / l_{1}$,

$\mathrm{C}_{12,13}=\mathrm{C}_{13,12}=\mathrm{C}_{5} \cdot l_{2}^{2} / l_{1} \cdot a_{1}-\mathrm{C}_{6} \cdot l_{2}^{2} / l_{1} \cdot a_{1}$

$\mathrm{C}_{13,13}=\mathrm{C}_{5} \cdot l_{2}^{2} / l_{1} \cdot a_{1}^{2}+\mathrm{C}_{6} \cdot l_{2}^{2} / l_{1} \cdot a_{1}^{2}$;
$\mathrm{K}_{1,1}=\mathrm{K}_{\mathrm{s}}, \mathrm{K}_{1,2}=-\mathrm{K}_{\mathrm{s}}, \mathrm{K}_{1,3}=\mathrm{K}_{\mathrm{s}}, \mathrm{e}_{1}, \mathrm{~K}_{1,4}=$ $\mathrm{K}_{\mathrm{s}} \cdot \mathrm{d}_{1}$

$\mathrm{K}_{2,1}=-\mathrm{K}_{\mathrm{s}}, \quad \mathrm{K}_{2,2}=4 \mathrm{~K}_{\mathrm{c}}+\mathrm{K}_{\mathrm{s}}, \quad \mathrm{K}_{2,3}=-\mathrm{K}_{\mathrm{s}}, \mathrm{e}_{1}$,

$\mathrm{K}_{2,4}=\mathrm{K}_{\mathrm{c}}\left(2 \mathrm{~d}_{3}-2 \mathrm{~d}_{2}\right)-\mathrm{K}_{\mathrm{s}} \cdot \mathrm{d}_{1}, \mathrm{~K}_{2,5}=-4 \mathrm{~K}_{\mathrm{c}}$, ,

$\mathrm{K}_{2,7}=\mathrm{K}_{\mathrm{c}}\left(2 \mathrm{~b}_{5}+2 \mathrm{~b}_{4}\right)$;

$\mathrm{K}_{3,1}=\mathrm{K}_{\mathrm{s}} \cdot \mathrm{e}_{1}, \mathrm{~K}_{3,2}=-\mathrm{K}_{\mathrm{s}} \cdot \mathrm{e}_{1}$,

$K_{3,3}=4 K_{c} \cdot e_{2}^{2}+K_{s} \cdot e_{1}^{2}, K_{3,4}=K_{s} \cdot e_{1} \cdot d_{1}$;

$\mathrm{K}_{4,1}=\mathrm{K}_{\mathrm{s}} \cdot \mathrm{d}_{1}$,

$\mathrm{K}_{4,2}=-\mathrm{K}_{\mathrm{s}} \cdot \mathrm{d}_{1}-2 \mathrm{~K}_{\mathrm{c}} \cdot \mathrm{d}_{2}+2 \mathrm{~K}_{\mathrm{c} 3} \cdot \mathrm{d}_{3}$,

$\mathrm{K}_{4,3}=\mathrm{K}_{\mathrm{s}} \cdot \mathrm{d}_{1} \cdot \mathrm{e}_{1}$,

$\mathrm{K}_{4,4}=\mathrm{K}_{\mathrm{s}} \cdot \mathrm{d}_{1}{ }^{2}+2 \mathrm{~K}_{\mathrm{c}}\left(\mathrm{d}_{2}{ }^{2}+\mathrm{d}_{3}{ }^{2}\right)$,

$\mathrm{K}_{4,5}=2 \mathrm{~K}_{\mathrm{c}}\left(\mathrm{d}_{2}-\mathrm{d}_{3}\right), \quad \mathrm{K}_{4,6}=-2 \mathrm{~K}_{\mathrm{c}} \cdot \mathrm{d}_{3} \cdot \mathrm{e}_{2}$,

$K_{4,7}=-2 K_{c} \cdot d_{2} \cdot b_{4}+2 K_{c} \cdot d_{3} \cdot b_{5}$;

$\mathrm{K}_{5,2}=-4 \mathrm{~K}_{\mathrm{c}}$,

$\mathrm{K}_{5,4}=-2 \mathrm{~K}_{\mathrm{c}} \cdot \mathrm{d}_{3}$

$\mathrm{K}_{5,5}=2 \mathrm{~K}_{\mathrm{f}}+2 \mathrm{~K}_{\mathrm{m}}+2 \mathrm{~K}_{\mathrm{r}}+4 \mathrm{~K}_{\mathrm{c}}$,

$\mathrm{K}_{5,6}=2\left(\mathrm{~K}_{\mathrm{f}}+\mathrm{K}_{\mathrm{m}}+\mathrm{K}_{\mathrm{r}}\right) \cdot \mathrm{a}_{1}+2 \mathrm{~K}_{\mathrm{c}} \cdot \mathrm{e}_{2}$,

$\mathrm{K}_{5,7}=-2 \mathrm{~K}_{\mathrm{f}}, \mathrm{b}_{1}+2 \mathrm{~K}_{\mathrm{m}}, \mathrm{b}_{2}+2 \mathrm{~K}_{\mathrm{r}}, \mathrm{b}_{3}-2 \mathrm{~K}_{\mathrm{c}}, \mathrm{b}_{4}$,

$\mathrm{K}_{5,8}=-2 \mathrm{~K}_{\mathrm{f}}, \quad \mathrm{K}_{5,10}=-2 \mathrm{~K}_{\mathrm{m}}, \mathrm{K}_{5,11}=2 \mathrm{~K}_{\mathrm{m}} \cdot \mathrm{a}_{1}$

$\mathrm{K}_{5,12}=-2 \mathrm{~K}_{\mathrm{r}}$;

$\mathrm{K}_{6,2}=-2 \mathrm{~K}_{\mathrm{c}}, \mathrm{e}_{2}, \mathrm{~K}_{6,3}=-2 \mathrm{~K}_{\mathrm{c}}, \mathrm{e}_{2}{ }^{2}$,

$\mathrm{K}_{6,4}=-2 \mathrm{~K}_{\mathrm{c}} \cdot \mathrm{e}_{2} \cdot \mathrm{d}_{3}$,

$\mathrm{K}_{6,5}=2\left(\mathrm{~K}_{\mathrm{f}}+\mathrm{K}_{\mathrm{m}}+\mathrm{K}_{\mathrm{r}}\right) \cdot \mathrm{a}_{1}+2 \mathrm{~K}_{\mathrm{c}} \cdot \mathrm{e}_{2}$,

$\mathrm{K}_{6,6}=2\left(\mathrm{~K}_{\mathrm{f}}+\mathrm{K}_{\mathrm{m}}+\mathrm{K}_{\mathrm{r}}\right) \cdot \mathrm{a}_{1}{ }^{2}+4 \mathrm{~K}_{\mathrm{c}} \cdot \mathrm{e}_{2}{ }^{2}$,

$\mathrm{K}_{6,7}=-2 \mathrm{~K}_{\mathrm{f}} \cdot \mathrm{a}_{1} \cdot \mathrm{b}_{1}-2 \mathrm{~K}_{\mathrm{m}} \cdot \mathrm{a}_{1} \cdot \mathrm{b}_{2}+2 \mathrm{~K}_{\mathrm{r}} \cdot \mathrm{a}_{1} \cdot \mathrm{b}_{3}-$ $2 \mathrm{~K}_{\mathrm{c}} \cdot \mathrm{e}_{2} \cdot \mathrm{b}_{4}$

$, \mathrm{K}_{6,8}=-2 \mathrm{~K}_{\mathrm{f}} \cdot \mathrm{a}_{1},, \mathrm{~K}_{6,12}=-2 \mathrm{~K}_{\mathrm{r}} \cdot \mathrm{a}_{1}$;

$\mathrm{K}_{7,2}=4 \mathrm{~K}_{\mathrm{c}}, \mathrm{b}_{4}, \mathrm{~K}_{7,4}=-2 \mathrm{~K}_{\mathrm{c}}, \mathrm{b}_{4} \cdot \mathrm{d}_{2}+2 \mathrm{~K}_{\mathrm{c}}, \mathrm{b}_{5} \cdot \mathrm{d}_{3}$,

$\mathrm{K}_{7,5}=-2 \mathrm{~K}_{\mathrm{f}}, \mathrm{b}_{1}+2 \mathrm{~K}_{\mathrm{m}}, \mathrm{b}_{2}+2 \mathrm{~K}_{\mathrm{r}}, \mathrm{b}_{2}-2 \mathrm{~K}_{\mathrm{c}}, \mathrm{b}_{4}-$ $2 \mathrm{~K}_{\mathrm{c}} \cdot \mathrm{b}_{5}$

,$\quad \mathrm{K}_{7,6}=-2 \mathrm{~K}_{\mathrm{f}} \cdot \mathrm{a}_{1} \cdot \mathrm{b}_{1}+2 \mathrm{~K}_{5} \cdot \mathrm{a}_{1} \cdot \mathrm{b}_{3}+2 \mathrm{~K}_{\mathrm{c}} \cdot \mathrm{e}_{2} \cdot \mathrm{b}_{4}$,

$\mathrm{K}_{7,7}=2 \mathrm{~K}_{\mathrm{f}} \cdot \mathrm{b}_{1}{ }^{2}+2 \mathrm{~K}_{\mathrm{m}} \cdot \mathrm{b}_{2}{ }^{2}+2 \mathrm{~K}_{\mathrm{r}} \cdot \mathrm{b}_{3}{ }^{2}+$

$2 \mathrm{~K}_{\mathrm{c}}, \mathrm{b}_{4}{ }^{2}+3 \mathrm{~K}_{\mathrm{c}} \cdot \mathrm{b}_{5}{ }^{2}$

, $\quad \mathrm{K}_{7,8}=2 \mathrm{~K}_{\mathrm{f}}, \mathrm{b}_{1}, \quad \mathrm{~K}_{7,10}=-2 \mathrm{~K}_{\mathrm{m}} \cdot \mathrm{b}_{2}$,

$\mathrm{K}_{7,11}=2 \mathrm{~K}_{\mathrm{m}}, \mathrm{b}_{2}, \mathrm{a}_{1}, \mathrm{~K}_{7,12}=-2 \mathrm{~K}_{\mathrm{r}}, \mathrm{b}_{3}$; 


$$
\begin{aligned}
& \mathrm{K}_{8,6}=-2 \mathrm{~K}_{\mathrm{f}} \cdot \mathrm{a}_{1} \text {, } \\
& \mathrm{K}_{8,7}=2 \mathrm{~K}_{\mathrm{f}}, \mathrm{b}_{1} \text {, } \\
& \mathrm{K}_{\mathrm{g}, 8}=2 \mathrm{~K}_{\mathrm{f}}+2 \mathrm{~K}_{\mathrm{wf}} \text {; } \\
& \mathrm{K}_{9,9}=2 \mathrm{~K}_{\mathrm{wf}} \cdot \mathrm{a}_{2}{ }^{2}+2 \mathrm{~K}_{1} \cdot \mathrm{a}_{1}{ }^{2} \quad \mathrm{~K}_{9,14}=-\mathrm{K}_{\mathrm{wf}} \cdot \mathrm{a}_{2}, \\
& \mathrm{~K}_{9,15}=\mathrm{K}_{\mathrm{wf}} \cdot \mathrm{a}_{2} \text {; } \\
& \mathrm{K}_{10,5}=-2 \mathrm{~K}_{\mathrm{m}} \text {, } \\
& \mathrm{K}_{10,7}=-2 \mathrm{~K}_{3} \cdot \mathrm{b}_{2} \text {, } \\
& \mathrm{K}_{10,6}=-2 \mathrm{~K}_{\mathrm{m}} \cdot \mathrm{a}_{1}, \\
& \mathrm{~K}_{10,10}=2 \mathrm{~K}_{\mathrm{wr}} \text {, } \\
& \mathrm{K}_{10,11}=-2 \mathrm{~K}_{3} \cdot \mathrm{a}_{1} \text {, } \\
& \mathrm{K}_{10,16}=-\mathrm{K}_{\mathrm{wr}} \text {, } \\
& \mathrm{K}_{10,17}=-\mathrm{K}_{\mathrm{wr}} \text {; } \\
& \mathrm{K}_{11,5}=2 \mathrm{~K}_{\mathrm{m}} \cdot \mathrm{a}_{1}, \quad \mathrm{~K}_{11,7}=2 \mathrm{~K}_{\mathrm{m}} \cdot \mathrm{b}_{2} \cdot \mathrm{a}_{1} \text {, } \\
& \mathrm{K}_{11,10}=-2 \mathrm{~K}_{\mathrm{m}} \cdot \mathrm{a}_{1} \text {, } \\
& \mathrm{K}_{11,11}=2 \mathrm{~K}_{\mathrm{m}} \cdot \mathrm{a}_{1}^{2}+2 \mathrm{~K}_{\mathrm{wr}} \cdot \mathrm{a}_{2}^{2} \text {, } \\
& \mathrm{K}_{11,16}=-\mathrm{K}_{\mathrm{wr}} \cdot \mathrm{a}_{2}, \mathrm{~K}_{11,17}=\mathrm{K}_{\mathrm{wr}} \cdot \mathrm{a}_{2} \text {; } \\
& \mathrm{K}_{12,5}=-2 \mathrm{~K}_{\mathrm{r}} \text {, } \\
& \mathrm{K}_{12,7}=-2 \mathrm{~K}_{\mathrm{r}} \cdot \mathrm{b}_{3} \text {, } \\
& \mathrm{K}_{12,6}=-2 \mathrm{~K}_{5} \cdot \mathrm{a}_{1} \text {, } \\
& \mathrm{K}_{12,12}=2 \mathrm{~K}_{\mathrm{wr}} \text {, } \\
& \text {, } \mathrm{K}_{12,18}=-2 \mathrm{~K}_{\mathrm{wr}} \text {; } \\
& \mathrm{K}_{13,13}=2 \mathrm{~K}_{\mathrm{wr}} \cdot \mathrm{a}_{2}{ }^{2}+2 \mathrm{~K}_{5} \cdot \mathrm{a}_{1}{ }^{2} \text {, } \\
& \mathrm{K}_{13,18}=-\mathrm{K}_{\mathrm{wr}} \cdot \mathrm{a}_{2}, \mathrm{~K}_{13,19}=-\mathrm{K}_{\mathrm{wr}} \cdot \mathrm{a}_{2} \text {; } \\
& \mathrm{K}_{14,8}=-\mathrm{K}_{\mathrm{wf}}, \mathrm{K}_{14,9}=-\mathrm{K}_{\mathrm{wf}} \cdot \mathrm{a}_{2}, \mathrm{~K}_{14,14}=\mathrm{K}_{\mathrm{wf}} ; \\
& K_{15,8}=-K_{w f}, K_{15,9}=K_{w f} \cdot a_{2}, K_{15,15}=K_{w f} ; \\
& \mathrm{K}_{16,10}=-\mathrm{K}_{\mathrm{wr}}, \quad \mathrm{K}_{16,11}=-\mathrm{K}_{\mathrm{wr}} \cdot \mathrm{a}_{2} \text {, } \\
& \mathrm{K}_{16,16}=\mathrm{K}_{\mathrm{wr}} \text {; } \\
& \mathrm{K}_{17,10}=-\mathrm{K}_{\mathrm{wr}}, \mathrm{K}_{17,11}=\mathrm{K}_{\mathrm{wr}}, \mathrm{a}_{2}, \mathrm{~K}_{17,17}=\mathrm{K}_{\mathrm{wr}} ; \\
& \mathrm{K}_{18,12}=-\mathrm{K}_{\mathrm{wr}}, \mathrm{K}_{18,13}=\mathrm{K}_{\mathrm{wr}}, \mathrm{a}_{2}, \mathrm{~K}_{18,18}=\mathrm{K}_{\mathrm{wr}} ; \\
& \mathrm{K}_{19,12}=-\mathrm{K}_{\mathrm{wr}}, \mathrm{K}_{19,13}=-\mathrm{K}_{\mathrm{wr}} \mathrm{a}_{2}, \mathrm{~K}_{19,19}=\mathrm{K}_{\mathrm{wr}} ;
\end{aligned}
$$

In which, $C_{s}$ and $K_{s}$ are the damping coefficient and stiffness of spring of driver seat; $\mathrm{C}_{\mathrm{c}}$ and $\mathrm{K}_{\mathrm{c}}$ are the damping and stiffness of each spring of cab suspension; $\mathrm{C}_{1}, \mathrm{C}_{2}$ are front suspension damping; $\mathrm{C}_{3 \sim 6}$ are the drive suspension coefficient; $\mathrm{C}_{\mathrm{e}}$ is the effective damping coefficient of drive axle suspension; $\mathrm{K}_{\mathrm{f}}$ is the stiffness of each spring of front axle suspension; $\mathrm{K}_{\mathrm{m}}$, and $\mathrm{K}_{\mathrm{r}}$ are defined for stiffness of every spring for the middle axle and the rear axle, respectively; $\mathrm{K}_{\mathrm{wf}}$ is also considered for the equivalent stiffness of each of the front tires, while $\mathrm{K}_{\mathrm{wr}}$ plays the same role for the tires of middle and rear wheels. Other variables and constants were illustrated in the previous sections.

The dynamic model developed here is 19 DoF which includes both the truck and 6 wheels. However, for analyzing under sinusoidal road surface excitation, the truck will be placed on the road. In this case, the motions of the 6 wheels is affected by the excitations of the road surface. Therefore, the 19 DoF model stiffness matrix and the proper model, simplifies to a 13 degrees of freedom system.

Let the excitations profile of road surface which applies to the wheels, be the sinusoid inputs as follow:

$w_{i}=A_{r} \sin \left(\omega_{d r} t+\right.$

$\left.\phi_{i}\right)$

$1,2, \ldots, 6$

$\omega_{d r}=2 \pi\left(\frac{v}{L}\right)$

Where $A_{r}$ is road roughness magnitude in meter, $\omega_{d r}$ is drive frequency in rad/s, $v$ is truck forward speed in $\mathrm{m} / \mathrm{s}, \mathrm{L}$ is Road surface wave length in meter and $\phi_{i}$ is the phase angle of the nth wheel in rad.

The equation of motion is:

$M \cdot \ddot{W}+C \cdot \dot{W}+K \cdot W=f(t)$

Where $\mathbf{M}$ is system mass matrix, $\mathbf{C}$ is damping matrix, $\mathrm{K}$ is stiffness matrix, $\ddot{W}$ is acceleration vector, $\dot{W}$ is velocity vector, $W$ is displacement vector, all of the 13 DoF truck model and $f(t)$ is road excitation vector, which has the form of:

$$
f(t)=\left[\begin{array}{c}
0 \\
\vdots \\
0 \\
k_{w 1} A_{r} \sin \left(\omega_{d r} t+\phi_{1}\right)+k_{w 2} A_{r} \sin \left(\omega_{d r} t+\phi_{2}\right) \\
k_{w 1} a_{2} A_{r} \sin \left(\omega_{d r} t+\phi_{1}\right)-k_{w 2} a_{2} A_{r} \sin \left(\omega_{d r} t+\phi_{2}\right) \\
k_{w 3} A_{r} \sin \left(\omega_{d r} t+\phi_{3}\right)+k_{w 4} A_{r} \sin \left(\omega_{d r} t+\phi_{4}\right) \\
k_{w 3} a_{2} A_{r} \sin \left(\omega_{d r} t+\phi_{3}\right)-k_{w 4} a_{2} A_{r} \sin \left(\omega_{d r} t+\phi_{4}\right) \\
k_{w 5} A_{r} \sin \left(\omega_{d r} t+\phi_{5}\right)+k_{w 6} A_{r} \sin \left(\omega_{d r} t+\phi_{6}\right) \\
k_{w 5} a_{2} A_{r} \sin \left(\omega_{d r} t+\phi_{5}\right)-k_{w 6} a_{2} A_{r} \sin \left(\omega_{d r} t+\phi_{6}\right)
\end{array}\right]_{(13 \times 1)}
$$

So as mentioned system matrices in Equation (8) is $13 \times 13$ and the vector $\mathrm{G}$ (gravity vector) does not appear 
because the initial positions are chosen as the system equilibrium positions.

Central difference method has been used for numerical solution and dynamic simulation, as bellow:

$\ddot{w} \approx \frac{w_{t+\Delta t}-2 w_{t}+w_{t-\Delta t}}{(\Delta t)^{2}}$

(9)

$\dot{w} \approx \frac{w_{t+\Delta t}-w_{t-\Delta t}}{2 \Delta t}$

The road profile that is used in the current simulation has a continuous sinusoidal variation as depicted in figure 5 .

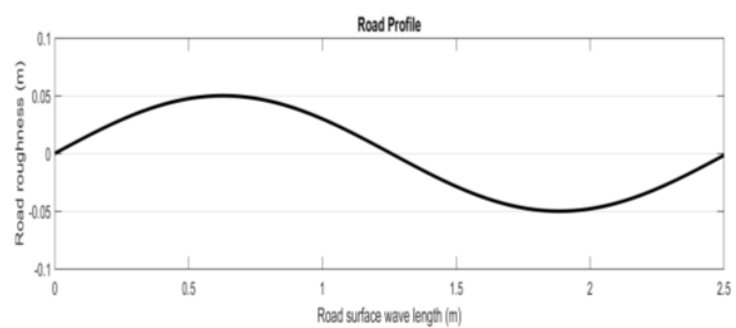

Figure 5. Profile of the road

In the current work, one drive frequency will be used to simulate a typical road condition. A high drive frequency is chosen which is $12 \mathrm{~Hz}$. The phase angle $\phi$ is selected for two cases. The first case is that the left steer wheel and the right steer wheel have no phase difference which means $\phi_{12}=0$ and the second case is that we have 90 degrees' phase lag for the right steer wheel compared to the left one and similarly this means that $\phi_{12}=\pi / 2$. For comparison, the two phase angle cases are depicted in Figure 6.
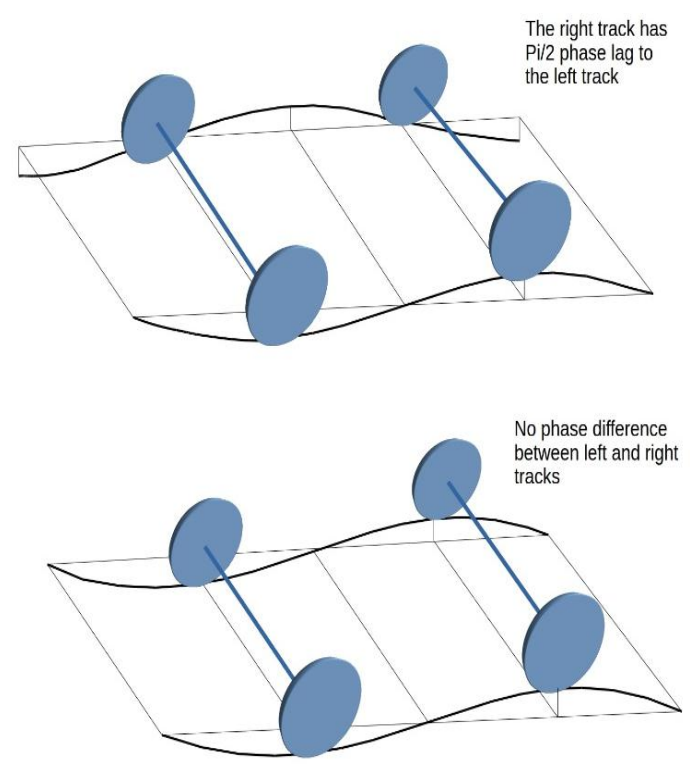

Figure 6. Excitation phase angle between the left and the right wheel for two cases

Subject to the assumed two phase angles, the phase angle of the nth wheel $\phi_{\mathrm{n}}$ is described as following:

$\phi_{1}=0$,

$\phi_{2}=\phi_{1}-\phi_{12}, \phi_{3}=\phi_{1}-\phi_{13}, \phi_{4}=\phi_{2}-\phi_{24}$

, $\phi_{5}=\phi_{1}-\phi_{15}, \phi_{6}=\phi_{2}-\phi_{26}$

Where $\phi_{12}, \phi_{13}, \ldots$ are the phase angle difference between wheel 1 and 2, wheel 1 and 3, etc. As following:

$$
\begin{aligned}
& \phi_{12}=0 \text { or } \frac{\pi}{2} \text { (depend on case of study); } \\
& \phi_{13}=\frac{2 \pi\left(b_{1}+b_{2}\right)}{L}, \\
& \phi_{15}=\frac{2 \pi\left(b_{1}+b_{3}\right)}{L}, \phi_{24}=\phi_{13}, \phi_{26}=\phi_{15}
\end{aligned}
$$

$\mathrm{L}$ is the road surface wave length in meter and $b_{1}, b_{2}, b_{3}$ are some geometric distance that can be seen in figure 4.

The drive frequency can be explained in two ways. For a specific road with a constant wave length of $\mathrm{L}$, the higher drive frequency is the higher speed the running truck, while the lower drive frequency, means that the truck is running at a lower speed. Similarly, if we consider that the truck speed is constant, the higher drive frequency means that the road surface possesses short wavelength characteristics, while, the lower drive frequency means that the road surface possesses long wavelength characteristics. So, the explanation of simulation results is strongly related to conditions and assumptions of the simulation. In this paper, we have considered that the road wave length is fixed and the truck speed is set to a high value of $30 \mathrm{~m} / \mathrm{s}$, and considering two different $\phi_{n}$ settings, gives us two cases in total, for both cases we have a high drive frequency, $A_{r}=0.05 \mathrm{~m}$ and $\omega_{\mathrm{dr}}=12 \mathrm{~Hz}$, for case $1, \phi_{12}$ is equal to zero and for case $2, \phi_{12}$ is equal to $\pi / 2$.

Finally, the simulation is performed by programming the equations in MATLAB software.

\section{RESULTS}

The following figures indicate the system time responses under two cases.

\section{Case 1:}




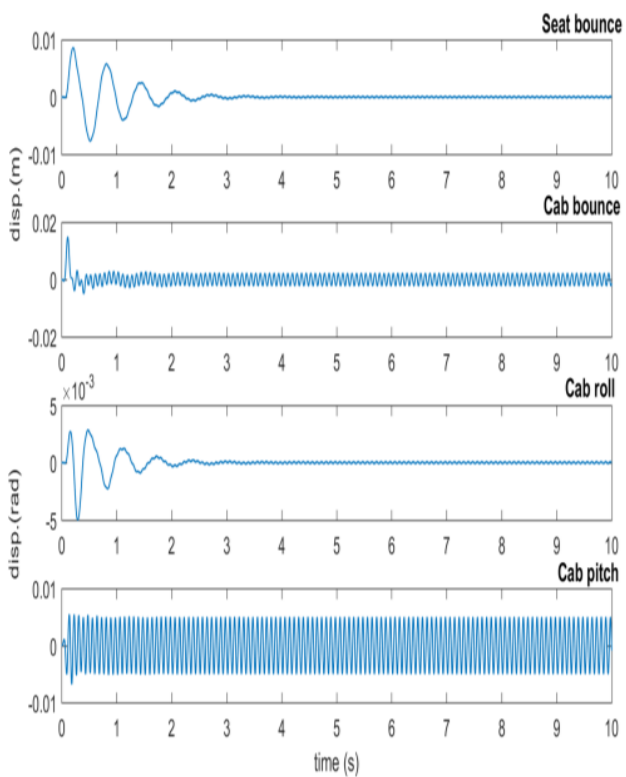

Figure 7. System time response for seat and cab in case 1

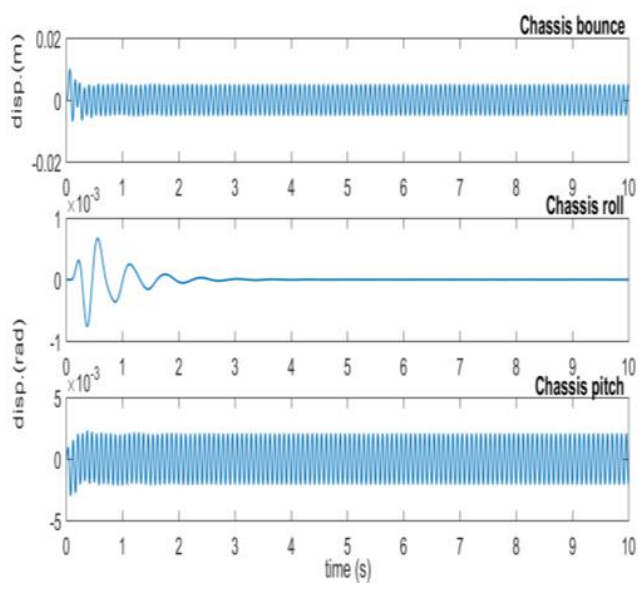

Figure 8. System time response for chassis in case 1
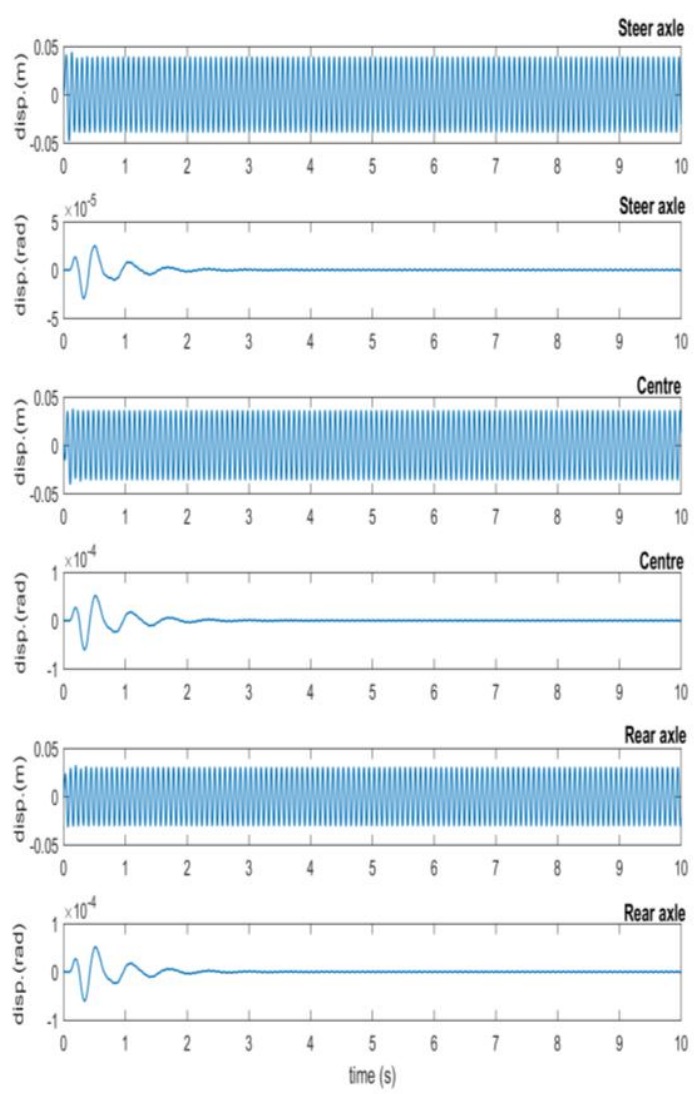

Figure 9. System time response for axles in case 1

In this case, the seat bounce and all of the roll DoFs take a relatively long time for settling to steady-state and hence shows a long transient state. However, the amplitudes of the roll motions are slight, this indicates insufficient damping especially about the roll axis.

\section{Case 2:}

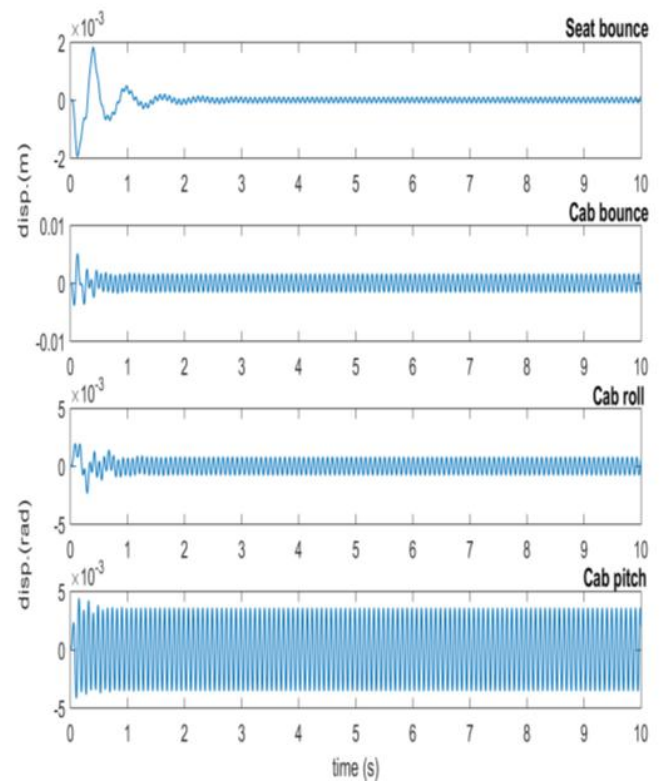


Figure 10. System time response for seat and cab in case 2

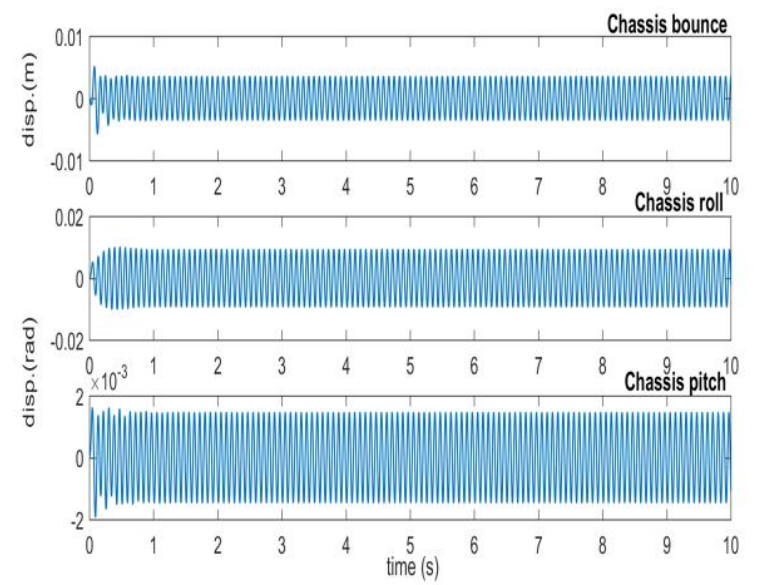

Figure 11. System time response for chassis in case 2
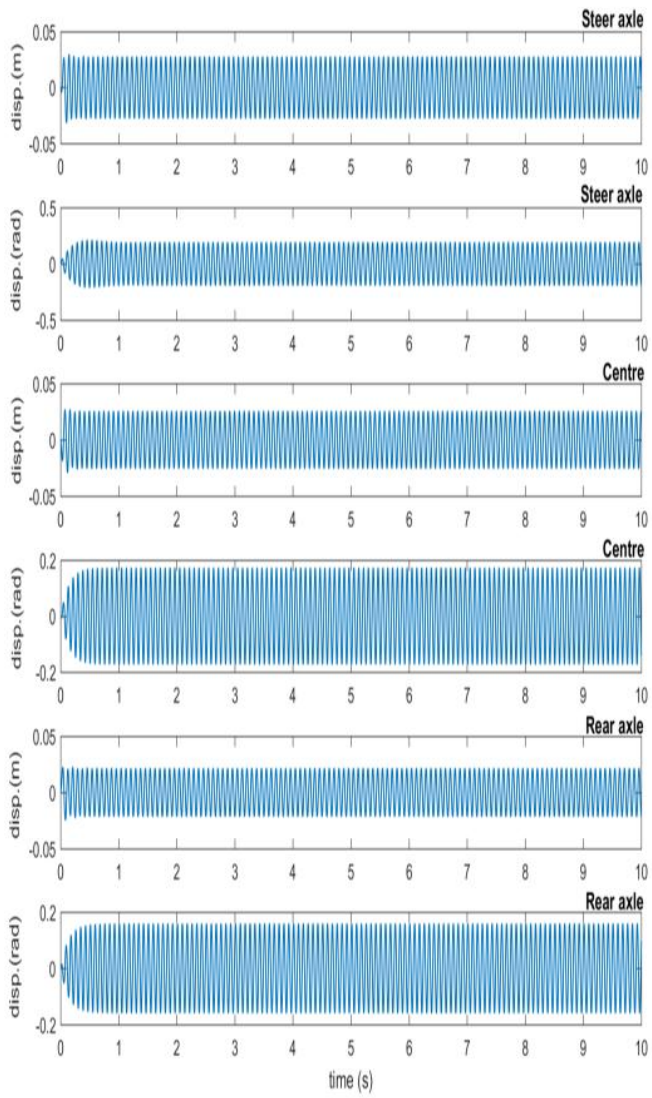

Figure 12. System time response for axles in case 2

In this case, the seat bounce, cab roll and chassis roll still have relatively long transient states. However, the 3 axles and the roll motions of the unsprang masses have been rapidly stabilized to the steady-state, because of the asymmetrical road excitations, it is clear that there are significant roll motions of the unsprang mass.

\section{CONCLUSION}

A 19-DoF system has been chosen in order to model a three-axle truck. Although this model, is a linear model, it has some unique features including the seat suspension, the cab suspension and the suspension geometry, which are vital for ride modeling of heavy vehicles but are often ignored. Physical properties of the truck are calculated using a model in Solidworks software. Lagrange equations are utilized for deriving equations of motion and the numerical central difference method is adopted to obtain the system responses subject to sinusoidal road excitations. Finally, system's time responses under two cases for the truck in high speed motion have been obtained, which are useful to know the vibrating component of the truck. Although some of them may only be effective to this particular truck model, they help better understanding the characteristics of this kind of vehicles and helps to understand the development of a more realistic nonlinear model.

\section{REFERENCES}

[1] Kadkhodapour, J. and S. Raeisi, Micro-macro investigation of deformation and failure in closedcell aluminum foams. Computational Materials Science, 2014. 83: p. 137-148.

[2] Rahmani, A., Mirmohammadi, A., Zeidi SMJ, Shojaei, S., (2015). Numerical Approach toward Calculation of vibration Characteristics of the Multi Axles Truck Using Lagrange Method, Journal of Modern Processes in Manufacturing and Production, vol. 4(1), pp. 57-64.

[3] Shojaei S., Zeidi SMJ, Rahmani, Mirmohammadi A., (2015). Analytical Analysis Approach to Study of the Vibration Characteristics of the Multi Axles Truck and its Validation. Proceedings of the International Conference in New Research of Industry and Mechanical Engineering.

[4] Hamedi, A., Malekmohammadi, I., Mansoori, A., \& Roshanaei, H. (2012). Energy Dissipation in Stepped Spillway Equipped with Inclined Steps Together with End Sill. Fourth International Conference on Computational Intelligence and Communication Networks. IEEE.

[5] Hamedi, A., Fuentes, H.R. (2016). New Relationship between a Vertical Gate Opening and Downstream Flow Stability: Experimental Development. World Environ. Water Resour. Congr,pp. 47-57.

[6] Hamedi, A., Fuentes, H.R. (2015). Comparative Effectiveness and Reliability of NEXRAD Data to Predict Outlet Hydrographs Using the GSSHA and HEC-HMS Hydrologic Models. World Environmental and Water Resources Congress, pp. 1444-1453.

[7] Hamedi, A., Hajigholizadeh, M., \& Mansoori, A. (2016). Flow Simulation and Energy Loss 
Estimation in the Nappe Flow Regime of Stepped Spillways with Inclined Steps and End Sill: A Numerical Approach. Civil Engineering Journal, 2(9), 426-437.

[8] Hamedi, A., Ketabdar, M. (2016). Energy Loss Estimation and Flow Simulation in the skimming flow Regime of Stepped Spillways with Inclined Steps and End Sill: A Numerical Model. International Journal of Science and Engineering Applications, 5(7), 399-407.

[9] Ketabdar, M. Hamedi, A. (2016). Intake Angle Optimization in 90-degree Converged Bends in the Presence of Floating Wooden Debris: Experimental Development. Florida Civil Engineering Journal, 2, 22-27.

[10] Watts GR (1987) Traffic-induced ground-borne vibrations in dwellings. Research Report 102, Transport and Road Research Laboratory, Crowthorne, Berkshire

[11] Hunt HEM (1991) Modelling of road vehicles for calculation of traffic-induced ground vibrations as a random process. J Sound Vib 144(1):41-51. doi:10.1016/0022-460X(91)90731-X

[12] Cebon D (1993) Interaction between heavy vehicles and roads. Warrendale (USA): Society of Automotive Engineers, SP 951: ISBN: 1-56091336-3

[13] Mamlouk MS (1997) General outlook of pavement and vehicle dynamics. J Transport Eng 123(6): 515-517. ISSN: 0733-947X

[14]Liu C, Herman R (1998) Road profiles, vehicle dynamics, and human judgement of serviceability of roads: spectral frequency domain analysis. J Transport Eng 124(2):106-111. doi:10.1061/(ASCE)0733-947X(1998)124:2(106)

[15] Noghrehabadi, Aminreza, Ehsan Izadpanahi, and Mohammad Ghalambaz. "Analyze of fluid flow and heat transfer of nanofluids over a stretching sheet near the extrusion slit." Computers \& Fluids 100 (2014): 227-236.

[16] Ghalambaz, M., E. Izadpanahi, A. Noghrehabadi, and A. Chamkha. "Study of the boundary layer heat transfer of nanofluids over a stretching sheet: Passive control of nanoparticles at the surface." Canadian Journal of Physics 93, no. 7 (2014): 725-733.

[17] Noghrehabadi, Aminreza, Mohammad Ghalambaz, Ehsan Izadpanahi, and Rashid Pourrajab. "Effect of magnetic field on the boundary layer flow, heat, and mass transfer of nanofluids over a stretching cylinder." Journal of Heat and Mass Transfer Research (JHMTR) 1, no. 1 (2014): 9-16.

[18] Noghrehabadi, Aminreza, Rashid Pourrajab, and Ehsan Izadpanahi. "Similarity Solution of Boundary Layer Flow over a Nanofluid-Saturated Stretching Cylinder." International Journal of Energy for a Clean Environment 16.1-4 (2015).

[19] Chamkha, A. J., Doostanidezfuli, A., Izadpanahi, E., \& Ghalambaz, M. (2017). Phase-change heat transfer of single/hybrid nanoparticles-enhanced phase-change materials over a heated horizontal cylinder confined in a square cavity. Advanced Powder Technology, 28(2), 385-397.

[20] Ghalambaz, M., Doostani, A., Izadpanahi, E., \& Chamkha, A. J. (2017). Phase-change heat transfer in a cavity heated from below: The effect of utilizing single or hybrid nanoparticles as additives. Journal of the Taiwan Institute of Chemical Engineers, 72, 104-115.

[21] Sheikholeslami ,A. and L. Azizi, (2010)."Safety Analysis of Constructed U-turns in the City of Tehran", Journal of Transportation Research 7 (223), 167-184.

[22] Azizi, L. and A. Sheikholeslami, (2013)."Safety effect of U-turn Conversions in Tehran: Empirical Bayes Observational Before and After Study and Crash Prediction Models", Journal of Transportation Engineering 139 (1),101-108.

[23] Sheikholeslami, A and L. Azizi, (2010) Observational Before-After Study of the Safety Effect of U-turns Conversions Using the Empirical Bayes Method, 15th International Conference on Road Safety CMRSC-XX

[24] Mardanpour, P., Izadpanahi, E., Rastkar, S., \& Hodges, D. H. (2017). Nonlinear Aeroelastic Gust Suppression and Engine Placement. Journal of Aircraft.

[25] Mardanpour, P., E. Izadpanahi, S. Rastkar, and D. H. Hodges. "Effects of Engine Placement on Nonlinear Aeroelastic Gust Response of HighAspect-Ratio Wings." In AIAA Modeling and Simulation Technologies Conference, p. 0576. 2017.

[26] Mardanpour, P., E. Izadpanahi, S. Rastkar, S. A. Fazelzadeh, and D. H. Hodges. "Geometricallyexact, fully intrinsic analysis of pretwisted beams under distributed follower forces." In 58th AIAA/ASCE/AHS/ASC Structures, Structural Dynamics, and Materials Conference, p. 1986. 2017.

[27] Azizi, L., MS. Iqbal and M.Hadi,(2018), Estimation of Freeway Platooning Measures Using Surrogate Measures Based on Connected Vehicle Data, Presented at 97st Annual Meeting of Transportation Research Board, Washington DC.

[28] Azizi,L., M.Hadi and Y.Xiao, (2018), Combination of Data from Multiple sources as Part of Dynamic Orgion Destination Matrix Estimation (ODME) Process.,Transportmetrica A: Transport Science.

[29] Melcer J (2006) Vehicle-road interaction, analysis in a frequency domain. Slovak J Civil Eng 3: 4852. ISSN: 1210-3896

[30] Dodds CJ, Robson JD (1973) The description of road surface roughness. J Sound Vib 31(2):175183. doi:10.1016/S0022-460X(73)80373-6

[31] Wambold JC, Defrain LE, Hegmon RR, Macghee K, Reichert J, Spangler EB (1981) State of the art 
of measurement and analysis of road roughness. Transport Res Rec 836: 21-29. ISSN: 0361-1981

[32] ISO 8608 (1995) Mechanical vibration, road surface profiles. Reporting of Measured Data

[33] Andren P (2006) Power spectral density approximations of longitudinal road profiles. Int $\mathbf{J}$ Veh Des 40(1/2/3):2-14. doi:10.1504/IJVD.2006.008450

[34] Elson MJ, Bennet JM (1995) Calculation of the power spectral density from surface profile data. Appl Opt 34:201-208. doi:10.1364/AO.34.000201

[35]Feng T, Yu-Fen H, Shun-Hsu T, Wes SJ (2006) Generation of random road profiles. CSME: B04001: 1373-1377

[36] Wong. J, Y., "Theory of ground vehicles". John Wiley \& Sons, Canada. (2001)

[37]Jazar, R., "Advanced Vibrations:A modern Approach", Springer, New York. (2013).

[38]Jazar, R., "Vehicle Dynamics: Theory and Application", Springer, New York. (2010).

[39] Gillespie, T, D., "Fundamentals of Vehicle Dynamics", SAE publishing group, United States of America. (1992).

[40] Yang, X., Zengcai, W., Weili, P., "Coordinated Control of AFS and DYC for Vehicle Handling and Stability Based on Optimal Guaranteed Cost Theory," Vehicle System Dynamics, 47, pp. 57-79 (2009).

[41] Zheng, S., Tang, H., Han, Z., Zhang, Y., "Controller Design for Vehicle Stability Enhancement," Control Engineering Practice, 14, pp. 1413-1421 (2006).

[42] A. Rahmani-Hanzaki, S. K. Saha, and P. V. M. Rao, An improved recursive dynamic modeling of a multibody system with spherical joint, Int, J, of Multibody system dynamics, 21, pp. 325-345, (2009).

[43] Tabatabaee, S,H,. "Integrated control to improve directional stability and maneuverability of the articulated heavy vehicle", PhD Thesis, K. N. Toosi University of Technology Faculty of Mechanical Engineering, (2013).

[44]Zeidi SMJ, Hoseini A, Rahmani A, 2017, "Study of vibration specifications of a three-axle truck using Lagrange method", International journal of science and engineering investigations.

[45]Zeidi, SMJ, Mahdi, M., (2015). Evaluation of the physical forces exerted on a spherical bubble inside the nozzle in a cavitating flow with an Eulerian/Lagrangian approach, European journal of physics, 136(6).

[46]Zeidi SMJ, Mahdi M, (2014). Investigation of viscosity effect on velocity profile and cavitation formation in Diesel injector nozzle, Proceedings of the 8th International Conference on Internal Combustion Engines.

[47]Zeidi SMJ, Mahdi M, (2015). Effects of nozzle geometry and fuel characteristics on cavitation phenomena in injection nozzles, Proceedings of the 22st Annual International Conference on Mechanical Engineering-ISME.

[48]Zeidi SMJ, Mahdi M, (2015). Investigation effects of injection pressure and compressibility and nozzle entry in Diesel injector nozzle's flow. J. Appl. Comp. Mech. 2 83-94.

[49] Azizi,L. , A.Sheikholeslami and F.B.Khalili. (2012) . Safety Analysis of Unconventional U-Turn Using Neural Network and Crash Prediction Model, 10th International Congress on Advances in Civil Engineering, 17-19 October 2012 Middle East Technical University, Ankara, Turkey, ACE.

[50]Zeidi SMJ, Hoseini P, Rahmani A, "Study of vibrations specifications of a three axle truck using Lagrange method", Journal of Modern Processes in Manufacturing and Production, vol. 6(1), pp. 8395. 